\title{
The Near-Death Experience as a Dissociative Phenomenon: An Empirical Assessment
}

\author{
Harvey J. Irwin, Ph.D. \\ University of New England
}

\begin{abstract}
Many commentators, particularly those working in a psychiatric framework, interpret the near-death experience as a dissociative phenomenon. The present study sought to assess the hypothesis that near-death experiencers are characterized by a dissociative response style engendered by severely traumatic childhood experiences. A postal survey of 121 Australian university students failed to identify a dissociative response style in experiencers, but these people did evidence a distinctive history of traumatic events in childhood. An attempt is made to reconcile these seemingly paradoxical findings.
\end{abstract}

In much of the psychiatric literature the near-death experience (NDE) is interpreted as an instance of dissociation. The purpose of this paper is to examine this view and to subject it to empirical investigation.

Some preliminary remarks on the nature of dissociation are appropriate. In the usual course of everyday life processes such as thoughts, memories, feelings, and sense of identity are relatively integrated. A particular train of thought, for example, may rekindle a distant memory that in turn may evoke various emotional reactions and may reaffirm the roots of the person we are. Dissociation may be defined as a structured separation of such mental processes (Spiegel and Cardeña,

Harvey J. Irwin, Ph.D., is Associate Professor of Psychology at the University of New England in Armidale, Australia. Reprint requests should be addressed to Dr. Irwin at the Department of Psychology, University of New England, Armidale, New South Wales 2351, Australia. 
1991). That is, feelings may be separated or "dissociated" from memories of specific incidents, and some memories may be kept separate or "repressed" from the flow of conscious thought. In pathological forms of dissociation such as multiple personality disorder, different clusters of memories and feelings may be maintained as separate "identities" within the one individual.

Although these processes lie at the heart of the so-called dissociative disorders (American Psychiatric Association, 1987), dissociation in itself is not pathological. Dissociative experiences are common in the general population (Ross, Joshi, and Currie, 1990). The capacity for dissociation evidently develops spontaneously in childhood as a normal process intrinsically associated with fantasy and imaginative ability (Putnam, 1991). Thus, young children commonly exhibit intense absorption in an activity, rapid attentional shifts, forgetfulness, and a capacity to take on another identity during play. Dissociative experiences in adolescence, however, tend to be transient and their incidence declines markedly between early adolescence and early adulthood (Ross, 1989). In normal adulthood, dissociative experiences are increasingly infrequent, their distribution across age being markedly skewed in the positive direction (Ross, Joshi, and Currie, 1990).

Some young children apparently capitalize on their heightened dissociative ability as the basis of a psychological coping mechanism. A rapidly expanding body of data (e.g., Sanders and Giolas, 1991) suggests that extraordinary childhood trauma is responsible for inducing this defensive use of dissociative processes. According to Spiegel (1986), although dissociation initially is used at the time of the trauma as a defense against (or attempt to adapt to) the associated pain and the sense of extreme vulnerability, the dissociative response subsequently becomes relied upon increasingly as a defense mechanism. In very general terms this course of development is construed as the origin of the dissociative disorders, in which the individual's mental processes become habitually fragmented. But the incidence of dissociative phenomena in otherwise normal adults is generally taken as an indication that many people develop a dissociative coping style without reaching the point where they fulfill the criteria for the diagnosis of a dissociative disorder.

The NDE frequently is spoken of, and even defined, as an instance of dissociation (e.g., Spiegel and Cardeña, 1991). Indeed, Serdahely (1992) recently has proposed that the NDE and multiple personality disorder are variants of the same phenomenological pattern. More typically this depiction of the NDE has been presented in terms of the concept of depersonalization, a psychiatric symptom that entails an altered sense 
of self. Among near-death researchers the first influential writer to adopt this approach was Russell Noyes. Particularly in his early writings Noyes (e.g., Noyes and Kletti, 1976) identified the NDE with depersonalization almost as a matter of definition, although he did support that definition by noting parallels between some phenomenological elements of the NDE and previously identified symptoms in depersonalized patients (e.g., Slater and Roth, 1969).

At the heart of the sense of self, however, is an awareness of one's identity. It is arguable that in phenomenological terms, a person having an NDE does not have an altered sense of identity. On the contrary, many near-death experiencers (NDErs) report that their impression of their self identity during the NDE was particularly lucid. What is altered in the NDE is the individual's association of self identity with their physical body, or more precisely, with current bodily sensations. In a life-threatening situation these sensations commonly would include anxiety symptoms and physical pain. During the NDE the experiencer is seemingly oblivious to such sensations. Indeed, the NDEr is oblivious also to the diverse somaesthetic and kinesthetic sensations that normally serve to remind one that phenomenologically speaking, the self is "in" the body. In psychiatric terminology these symptoms are most aptly described as dissociation rather than simply as depersonalization. From this perspective, sense of identity and mental imagery appear to be dissociated from physical sensations and (the physical concomitants of) emotions. In any event it is fair to say that a number of researchers working in a psychiatric framework, including those who prefer the terminology of depersonalization, essentially are interpreting the NDE as a dissociative phenomenon.

Under the dissociation model it might be predicted that many NDErs are characterized by a dissociative coping style. In other words, NDErs may react to threatening circumstances with dissociative behavior because they were prompted to develop such a coping style in childhood. Little attention seems to have been devoted to a direct empirical assessment of this hypothesis. The recent study by Kenneth Ring and Christopher Rosing (1990) nevertheless is most pertinent. A comparison between NDErs and control participants was undertaken on an inventory of items concerning childhood abuse and trauma. Ring and Rosing reported a significant difference between the groups on all dimensions surveyed, namely physical abuse, psychological abuse, sexual abuse, neglect, and negative home atmosphere. These researchers interpret their data to imply that as a result of a history of childhood abuse, NDErs develop a "dissociative response style" (p. 231), enabling them to "tune into" alternate, nonordinary realities (p. 232). 
Although Ring and Rosing's notion of the nonordinary reality of an NDE is a moot inference, their findings do offer a degree of support for the dissociation approach to NDEs. There are, however, some limitations to their study. One problem is that the surveyed dimensions of childhood trauma may well be intercorrelated. Although Ring and Rosing acknowledged this point, they did not utilize a statistical procedure such as discriminant analysis to overcome the problem, apparently opting instead for a series of univariate tests. It is possible, therefore, that their two groups in effect did not differ on all aspects of trauma. By way of illustration, if "neglect" is a common element in many other childhood traumas, the groups may differ on this factor but not on other factors once the contribution of neglect has been partialled out. A second limitation of the study is that it surveyed a comparatively limited range of childhood trauma. Use of a broader index of trauma not only would offer the opportunity for a constructive replication of Ring and Rosing's findings, but also might reveal other categories of trauma that have a bearing on NDErs' alleged dissociative coping style. Additionally, there is scope for undertaking a direct test of the dissociation hypothesis by assessing NDErs' relative proneness to dissociative experiences.

In seeking to address these limitations of the previous research the present study sought to assess further a dissociation approach to the NDE.

\section{Method}

\section{Participants}

The study was undertaken as part of a larger postal questionnaire survey of adults enrolled in an off-campus Introductory Psychology course taught through the University of New England in Australia. Students in this course generally are of mature age; most are in paid employment, some are homemakers. This group thus may be deemed to be more similar to the general population than are typical undergraduate psychology students.

Survey forms were mailed to the 152 members of the class. Completed forms were returned by 121 people, a participation rate of 80 percent. The net sample comprised 32 men and 89 women, ranging in age from 19 to 72 years $($ mean $=37.1$, median $=36$ ). 


\section{Materials}

The survey inventory included three questionnaires. One was a brief form requesting details of gender and age, and asking if the respondent had had an NDE, that is, a transcendent state of consciousness or awareness experienced under life-threatening circumstances. The other two questionnaires related to dissociation and to childhood trauma.

The measure of proneness to dissociation was Kevin Riley's (1988) Questionnaire of Experiences of Dissociation (QED). The QED comprises 26 dichotomous (true/false) items tapping experiences of dissociative phenomena. Scores thus may range from 0 to 26, with higher scores signifying a greater range of dissociative experiences acknowledged by the respondent. The QED has been standardized on normal samples. The scale's reliability is satisfactory, and the measure has been validated both through application to clinical samples with dissociative disorders (Riley, 1988) and by factor analytic comparison to another similar measure (Ray, June, Turaj, and Lundy, 1992).

Childhood trauma was indexed by the Survey of Traumatic Childhood Events (STCE) (Council and Edwards, 1987). The 30 items of the STCE tap childhood trauma of eleven types: intrafamilial sexual abuse, extrafamilial sexual abuse, intrafamilial physical abuse, loss related to a friend, loss related to the family, isolation, personal illness or accident, parental divorce/separation and abortion/miscarriage, assault, loss of the home, and robbery. Responses are made on a fivepoint scale ( 1 = "none", to 5 = "more than ten"). For each of the eleven subscales a score is computed by summation across items, with high scores attesting to a high incidence of the given class of childhood trauma. No psychometric data for the STCE are yet available.

\section{Procedure}

An informed consent form was attached to the front of the inventory mailed to potential participants. This sheet explained the objective of the study, drawing attention to the personal nature of items in the STCE and stressing that participation was voluntary and confidential. An appeal was made to participants to respond as spontaneously and openly as possible.

Participants returned their completed questionnaires in a stamped envelope supplied by the researcher. A substantial majority of 
inventories were returned within a few weeks of the original distribution, but occasional returns still were being received some three months later. At the latter date a decision was made to regard the process of data collection as complete, and statistical analysis then was undertaken.

\section{Results}

For the purpose of statistical analysis, participants were allocated to one of two groups on the basis of their response to the item about a past NDE. Ten students responded affirmatively to this question; they were designated as NDErs. The remaining 111 participants served as a control group. The incidence of NDErs in the sample thus was 8 percent, a result comparable to findings of previous research (Locke and Shontz, 1983). It may be noted that all NDErs in the sample had encountered their experience at the age of 18 years or older; that is, the NDE did not occur in childhood and thus its circumstances can not be regarded as one of the childhood trauma indexed by the STCE.

Mean scores on each of the research measures are given for each group in Table 1.

\section{Table 1}

Mean Scores of each Group on Measures of Dissociation (QED) and Childhood Trauma (STCE)

\begin{tabular}{lcc}
\hline Scale & $\begin{array}{c}\text { NDErs } \\
(n=10)\end{array}$ & $\begin{array}{c}\text { Controls } \\
(n=111)\end{array}$ \\
\hline QED & 10.40 & 8.96 \\
STCE scales & & \\
$\quad$ Intrafamilial sexual abuse & 3.10 & 3.87 \\
Intrafamilial physical abuse & 17.30 & 15.46 \\
Loss related to friend & 4.20 & 3.45 \\
Extrafamilial sexual abuse & 6.20 & 4.42 \\
Loss related to family & 3.90 & 4.79 \\
Isolation & 2.90 & 2.60 \\
Personal illness or accident & 2.60 & 2.60 \\
Parental divorce or separation; & & \\
$\quad$ abortion or miscarriage & 2.40 & 2.47 \\
Assault & 4.10 & 2.63 \\
Loss of home & 5.30 & 4.64 \\
Robbery & 1.00 & 1.05 \\
\hline
\end{tabular}


Although the mean QED score for NDErs was slightly higher than that for the control participants, the difference between groups was not significant $(t=0.96, d f=119)$. That is, there is no evidence here that NDErs as a group are especially prone to dissociation.

A discriminant function analysis was performed using the eleven subscales of the STCE as predictors of membership in the two groups. The discriminant function was highly significant (chi-squared $=27.24$, $\mathrm{df}=10, \mathrm{p}<.005$ ). Dimensions of childhood trauma contributing substantially to the prediction of group membership were assault (canonical loading $=.65$ ), extrafamilial sexual abuse (.41), and loss related to a friend (.37).

\section{Discussion}

The lack of any evident difference between NDErs and others in proneness to dissociation is an interesting result. It suggests that experiencers are not habitually inclined to use a dissociative response style. In this respect the findings do not offer support for Ring and Rosing's (1990) interpretations of their data, nor indeed for the claim of Serdahely (1992) that the NDE and multiple personality disorder are variants of the same phenomenological pattern. This result does warrant replication in a larger sample of NDErs, but given that for my sample the value of $t$ was less than 1, the prospects for establishing a significant relationship seem remote.

At the same time the data clearly support the broad findings of Ring and Rosing (1990), that NDErs as a group tend to have had a relatively traumatic childhood. Because the trauma indexed by the STCE occurred before the NDE, the higher level of traumatic events in the NDErs' childhood is not a mere confound of the circumstances of the NDE itself. The results of the discriminant function analysis therefore attest to a significant developmental difference between experiencers and nonexperiencers.

Contrary to the findings of Ring and Rosing, however, the data suggest that some traumatic events are much more important than others in discriminating between the two groups of people. The factors contributing most strongly to the discrimination were assault by a stranger, extrafamilial sexual abuse, and separation by injury, illness, or death from a close personal friend. One possibly cogent characteristic of these specific types of traumatic event is their episodic and unpredictable nature. Some of the factors that did not contribute to the discriminant function, on the other hand, commonly tend to be persistent 
and relatively predictable in the life of the traumatized child, such as intrafamilial sexual abuse, intrafamilial physical abuse, and living in isolation.

This characteristic of the discriminating trauma in NDErs' childhood suggests a means of reconciling the STCE results with those for the QED. It is possible that childhood events encountered by NDErs in fact do not induce a general dissociative defense style that is used whenever the person has difficulty in coping with prevailing circumstances. Rather, severe and unpredictable episodic trauma may engender a tendency to use a dissociative response in specific situations marked by a highly stressful but unforeseen threat. In other words, the dissociative defense may be relatively situation-specific. This version of the dissociation model would account for the occurrence of an NDE when such an individual is in a life-threatening context and for the paradoxically "normal" level of dissociative behavior in most other settings. Perhaps other researchers will be able to replicate my findings and extend my speculations through a detailed formulation of a dissociation model of the NDE.

\section{References}

American Psychiatric Association. (1987). Diagnostic and statistical manual of mental disorders (3rd edition, revised). Washington, DC: American Psychiatric Press.

Council, J.R., and Edwards, P.W. (1987). Survey of traumatic childhood events. Unpublished measure. Fargo, ND: North Dakota State University.

Locke, T.P., and Shontz, F.C. (1983). Personality correlates of the near-death experience: A preliminary study. Journal of the American Society for Psychical Research, 77, 311318.

Noyes, R., and Kletti, R. (1976). Depersonalization in the face of life-threatening danger: A description. Psychiatry, 39, 19-27.

Putnam, F.W. (1991). Dissociative disorders in children and adolescents: A developmental perspective. Psychiatric Clinics of North America, 14, 519-531.

Ray, W.J., June, K., Turaj, K., and Lundy, R. (1992). Dissociative experiences in a college age population: A factor analytic study of two dissociation scales. Personality and Individual Differences, $13,417-424$.

Riley, K.C. (1988). Measurement of dissociation. Journal of Nervous and Mental Disease, 176, 449-450.

Ring, K., and Rosing, C.J. (1990). The Omega Project: An empirical study of the NDEprone personality. Journal of Near-Death Studies, 8, 211-239.

Ross, C.A. (1989). Multiple personality disorder: Diagnosis, clinical features, and treatment. New York: Wiley.

Ross, C.A., Joshi, S., and Currie, R. (1990). Dissociative experiences in the general population. American Journal of Psychiatry, 147, 1547-1552.

Sanders, B., and Giolas, M.H. (1991). Dissociation and childhood trauma in psychologically disturbed adolescents. American Journal of Psychiatry, 148, 50-54.

Serdahely, W.J. (1992). Similarities between near-death experiences and multiple personality disorder. Journal of Near-Death Studies, 11, 19-38. 
Slater, E., and Roth, M. (1969). Clinical psychiatry (3rd ed.). Baltimore, MD: Williams and Wilkins.

Spiegel, D. (1986). Dissociating damage. American Journal of Clinical Hypnosis, 29, 123-131.

Spiegel, D., and Cardeña, E. (1991). Disintegrated experience: The dissociative disorders revisited. Journal of Abnormal Psychology, 100, 366-378. 